\title{
Article \\ Metabolomic and Transcript Analysis Revealed a Sex-Specific Effect of Glyphosate in Zebrafish Liver
}

\author{
Christian Giommi ${ }^{1}\left(\mathbb{D}\right.$, Claudia Ladisa $^{2}$, Oliana Carnevali $^{1,3}\left[\right.$, Francesca Maradonna ${ }^{1,3, * \mathbb{C}}$ \\ and Hamid R. Habibi ${ }^{2}$ (D) \\ 1 Dipartimento Scienze della Vita e dell'Ambiente, Università Politecnica delle Marche, Via Brecce Bianche, \\ 60131 Ancona, Italy; c.giommi@pm.univpm.it (C.G.); o.carnevali@univpm.it (O.C.) \\ 2 Department of Biological Sciences, University of Calgary, Calgary, AB T2N 1N4, Canada; \\ claudia.ladisa1@ucalgary.ca (C.L.); habibi@ucalgary.ca (H.R.H.) \\ 3 INBB - Consorzio Interuniversitario di Biosistemi e Biostrutture, 00136 Roma, Italy \\ * Correspondence: f.maradonna@univpm.it
}

Citation: Giommi, C.; Ladisa, C.; Carnevali, O.; Maradonna, F.; Habibi, H.R. Metabolomic and Transcript Analysis Revealed a Sex-Specific Effect of Glyphosate in Zebrafish Liver. Int. J. Mol. Sci. 2022, 23, 2724 https://doi.org/10.3390/ ijms23052724

Academic Editor: Kanako Hayashi

Received: 31 January 2022

Accepted: 28 February 2022

Published: 1 March 2022

Publisher's Note: MDPI stays neutral with regard to jurisdictional claims in published maps and institutional affiliations.

Copyright: (C) 2022 by the authors. Licensee MDPI, Basel, Switzerland. This article is an open access article distributed under the terms and conditions of the Creative Commons Attribution (CC BY) license (https:// creativecommons.org/licenses/by/ $4.0 /)$.
Abstract: Glyphosate is a component of commonly used herbicides for controlling weeds in crops, gardens and municipal parks. There is increasing awareness that glyphosate-based herbicides, in addition to acting on plants, may also exert toxicity in wildlife and humans. In this study, male and female adult zebrafish were exposed to $700 \mu \mathrm{g} / \mathrm{L}$ of glyphosate (GLY), for 28 days. We used the metabolomic approach and UHPLC-ESI-MS to analyze liver samples to investigate the adverse effects of glyphosate on hepatic metabolism. The impact of GLY was found to be sex-specific. In female, GLY exposure affected purine metabolism by decreasing the levels of AMP, GMP and inosinic acid, consequently increasing uric acid levels with respect to the control (CTRL). Exposure to GLY also caused a decrease of UMP levels in the pyrimidine metabolism pathway. In male, GLY exposure decreased the aminoadipic acid within the lysine degradation pathway. Transcript analysis of genes involved in stress response, oxidative stress and the immune system were also performed. Results demonstrated an increased stress response in both sexes, as suggested by higher $n r 3 c 1$ expression. However, the $h s p 70.2$ transcript level was increased in female but decreased in male. The results demonstrated reduced sod1, sod2, and gpx1a in male following exposure to GLY, indicating an impaired oxidative stress response. At the same time, an increase in the cat transcript level in female was observed. mRNA levels of the pro-inflammatory interleukins litaf and cxcl8b.1 were increased in female. Taken together, the results provide evidence of disrupted nucleotide hepatic metabolism, increased stress inflammatory response in female and disruption of oxidative stress response in male.

Keywords: Danio rerio; metabolomic; liver; glyphosate; Roundup ${ }^{\circledR}$; purine metabolism; oxidative stress

\section{Introduction}

In both plants and microorganisms, the mechanism of action of the non-selective herbicide glyphosate (GLY), which is an amino phosphonic analogue of the amino acid glycine, relies on the inhibition of the enzyme 5-enolpyruvylshikimate-3-phosphate synthase (EPSPS) activity, leading to reduced aromatic amino acid synthesis [1]. To improve absorption, glyphosate isopropylamine salt is used in combination with polyethoxylated tallow amine (POEA) which is the most utilized form of coadjutants. These formulations are sold under the trade name of Roundup ${ }^{\circledR}$, in which the active ingredient is indicated as glyphosate acid equivalent (a.e.) and could be present at different concentrations. Currently, this herbicide is used extensively to eliminate weeds in conjunction with the use of genetically modified GLY-resistant plants. These formulations are also found in municipal parks as well as public and private gardens. There is evidence that this compound can reach surface waters via direct applications and runoffs. Limits for the presence of GLY in drinking water range from the highest levels in Australia $(1000 \mu \mathrm{g} / \mathrm{L})$ and USA $(700 \mu \mathrm{g} / \mathrm{L})$ to lower levels in Canada $(280 \mu \mathrm{g} / \mathrm{L})$ and Europe $(0.1 \mu \mathrm{g} / \mathrm{L})$ [2]. Despite the claim that GLY-based herbicides 
only target plant-specific enzymatic pathway, various studies demonstrated adverse effects of this contaminant in both vertebrate and invertebrates [3-5], as well as in wildlife and aquatic species [6-10], where this contaminant, alone or in formulation, induced oxidative stress and DNA damage. These observations are raising concern about public health since GLY can be detected in the urine of humans [11]. Thus we used a metabolomic- and transcriptomic-based approach to investigate the toxicity of GLY alone and the equivalent concentration of GLY in combination with coadjutants present in Roundup ${ }^{\circledR}$ in zebrafish.

\section{Results}

\subsection{Metabolomic Characterization of the Liver}

A metabolomic approach was used to analyze GLY-induced changes of hepatic metabolism. Data generated from ultra-high-performance liquid chromatography coupled with high-resolution full-scan mass spectroscopy (UHPLC-MS) were visualized on MAVEN software as peaks with intensity corresponding to the concentration of metabolites in the samples. MAVEN, together with a standard library of mass to charge $(\mathrm{m} / \mathrm{z})$ and retention times of metabolites, allowed the identification of 71 metabolites in zebrafish liver extracts, with levels differing between CTRL and GLY groups. The metabolic profile was studied by means of multivariate analysis and visualization techniques, including principal component analysis (PCA) and partial-least squares-discriminant analysis (PLS-DA). Seven extracted pools of female livers and four extracted pools of male livers were used to perform PCA with the quality control group $(\mathrm{QC}, n=4)$, to investigate the presence of outliers and assess the dataset reliability. In this regard, the PCA scatter plot demonstrated a strong cluster formation for the QC group (Figure 1a,b) in females and males, despite lacking a clear separation between the groups.

a)

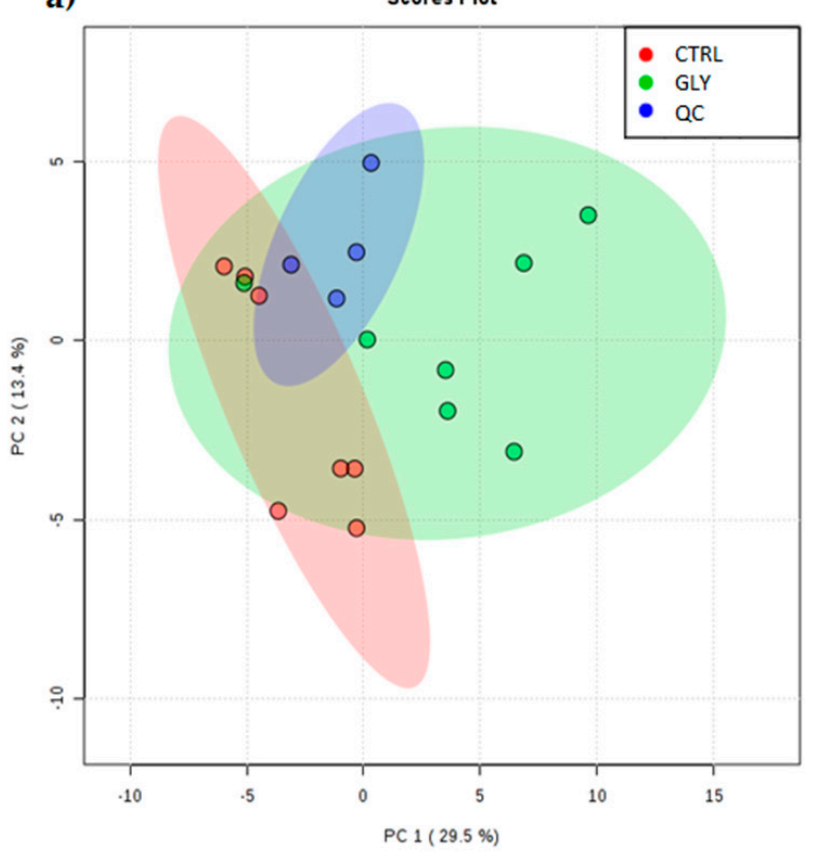

b)

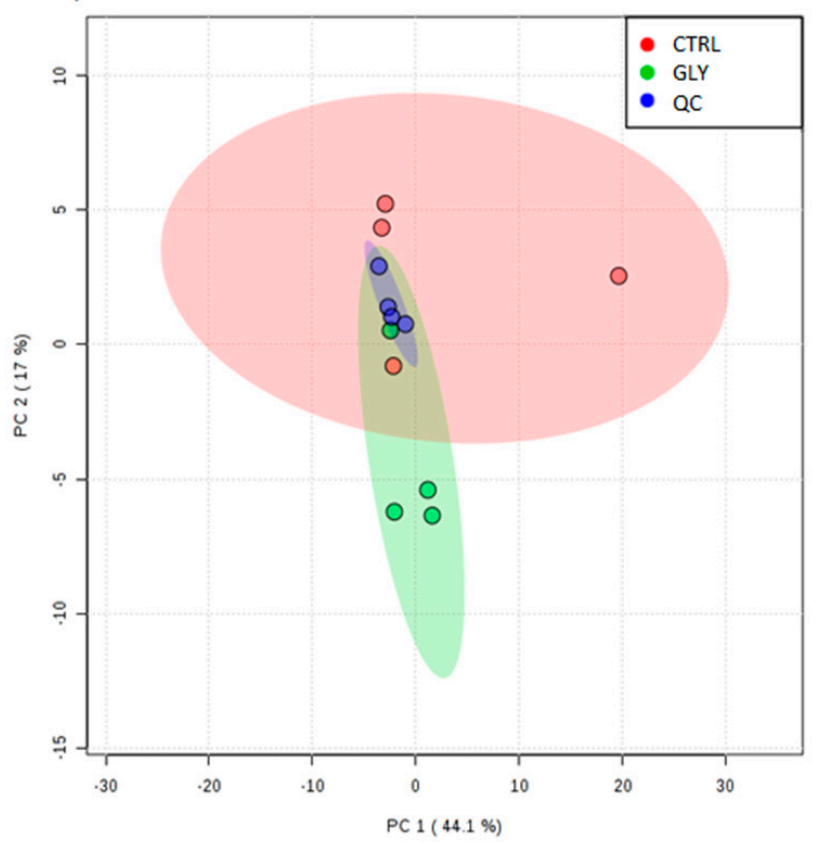

Figure 1. PCA score plot of CTRL and GLY metabolic profile in female and male livers. PCA score scatter plot of CTRL (red) vs. GLY (green) and QC (blue) in (a) female and (b) male livers. The abscissa axis shows the PC1 while the ordinate axis shows the PC2; the percentage of total variance for each PC is shown in parentheses. All samples are comprised within the $95 \%$ confidence interval of their respective group showing the absence of outliers. QC samples show strong cluster formation.

PLS-DA analysis was then performed to investigate differences between treatment groups in both male and female fish. The quality parameters (R2 and Q2) and $p$-values for the built models, were estimated for the analysis by the SIMCA program. Since the 
good quality of a built model is set for R2 and Q2 > 0.5, it can be assumed that a good and significant separation was observed between the CTRL and GLY-exposed groups in females $(p<0.003 ; \mathrm{R} 2=0.915, \mathrm{Q} 2=0.791)$ (Figure 2a,c). However, in males, the cluster separation was not significantly different between the CTRL and GLY-exposed groups $(p<0.572$; $\mathrm{R} 2=0.91, \mathrm{Q} 2=0.578$ ) despite the good quality of the model (Figure $2 \mathrm{~b}, \mathrm{~d}$ ). Hierarchical analysis revealed separation of treatment groups in both sexes (Figure 2e,f).

a)

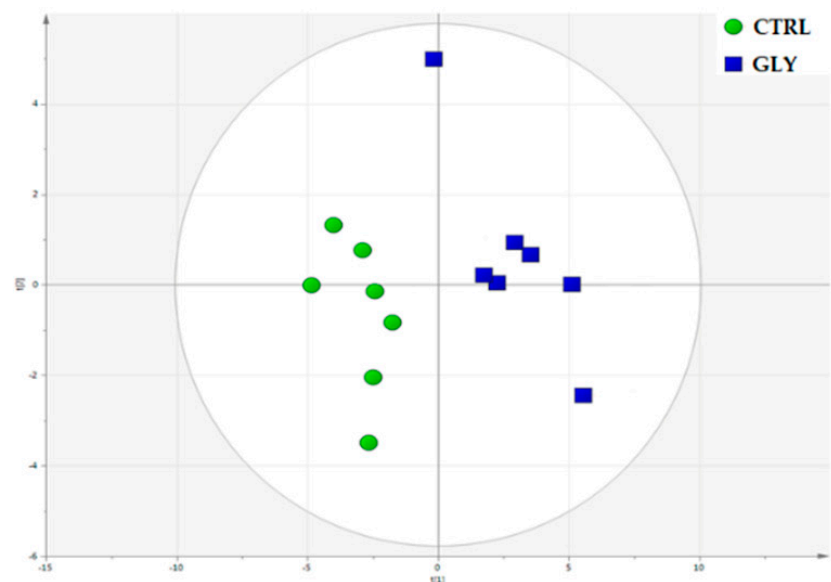

c)

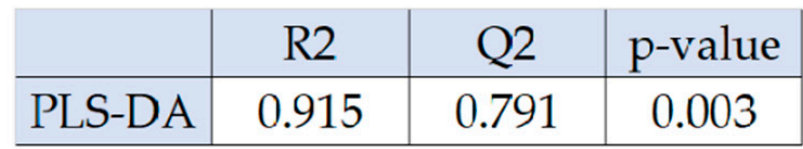

e)

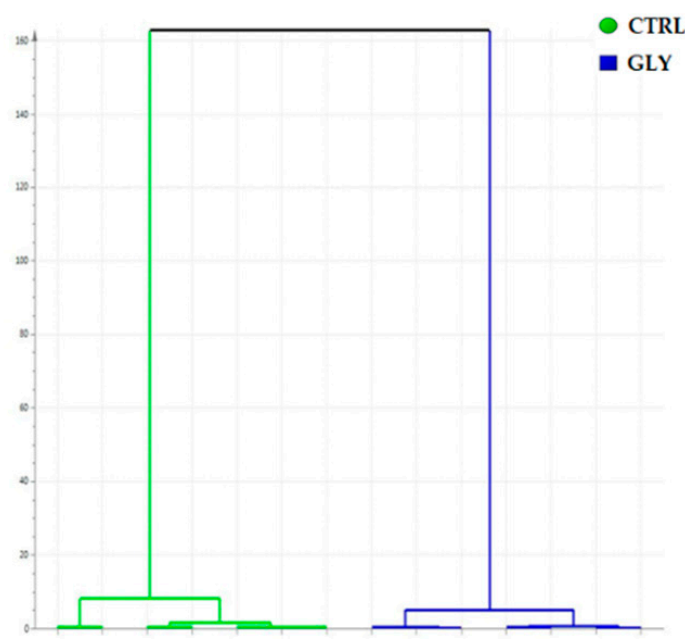

b)

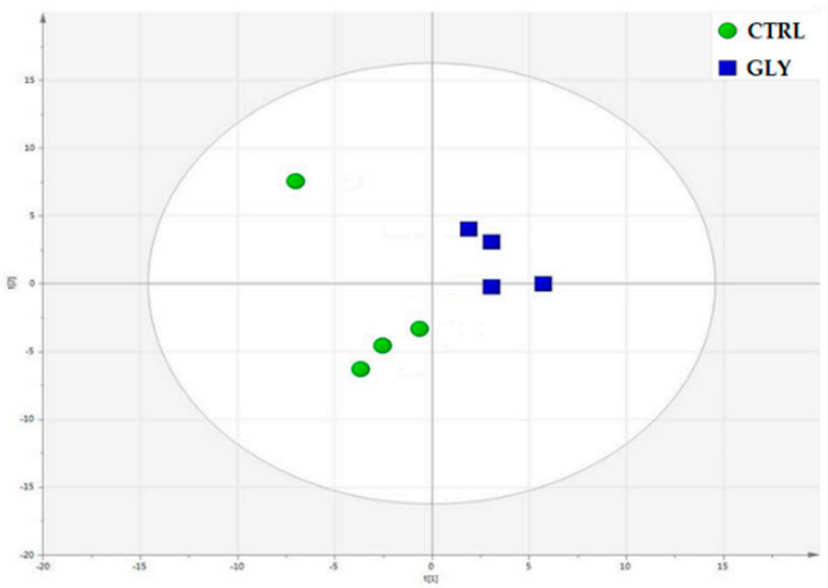

d)

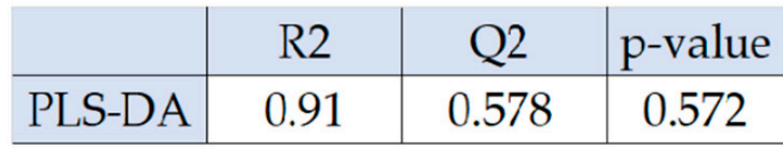

f)

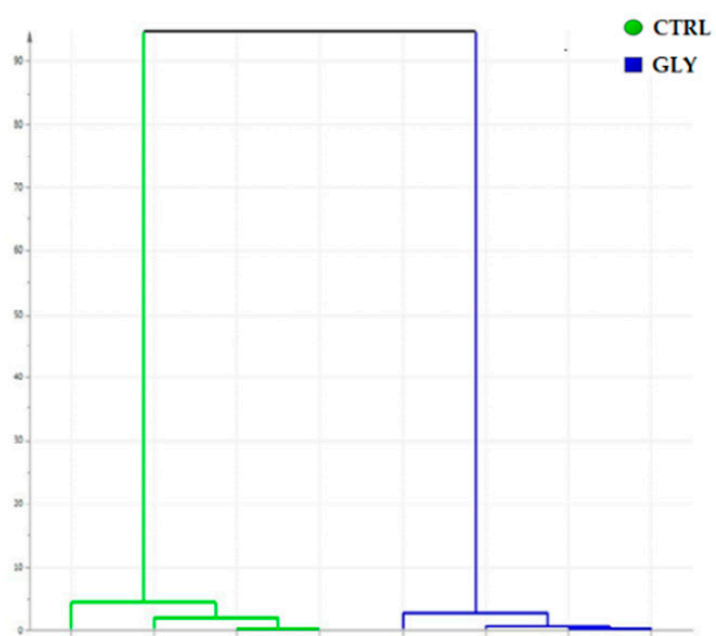

Figure 2. PLS-DA score plot and hierarchical analysis in CTRL and GLY females and males. PLS-DA score scatter plot of CTRL (green) vs. GLY (blue) in (a) females and (b) males. (c,d) Quality parameters (R2 and Q2) and $p$-value of the two PLS-DA-built models in females and males, respectively. Hierarchical analysis of CTRL (green) vs. GLY (blue) in (e) females and (f) males.

The variable importance in projection (VIP) score was used to determine the importance of metabolites in the built models. A VIP score $\geq 1$ is usually considered as threshold for the selection of individual metabolites used for the SIMCA PLS-DA model building and are then used for further analysis (Table $1 a, b)$. 
Table 1. VIP score of metabolites found with PLS-DA analysis.

\begin{tabular}{|c|c|c|c|}
\hline \multicolumn{2}{|l|}{ (a) } & \multicolumn{2}{|l|}{ (b) } \\
\hline Metabolites & VIP (12) & Metabolites & VIP (25) \\
\hline N-Acetyl-L-methionine & 1.3711 & Hippuric acid & 1.98682 \\
\hline Docosahexaenoic acid & 1.29572 & Aminoadipic acid & 1.86273 \\
\hline Sucrose & 1.26731 & Docosahexaenoic acid & 1.68523 \\
\hline L-Homoserine & 1.25124 & N-Acetylleucine & 1.66798 \\
\hline Azelaic acid & 1.23396 & Cytidine monophosphate & 1.65299 \\
\hline Hypotaurine & 1.23293 & Uracil & 1.64123 \\
\hline Adenosine monophosphate & 1.13408 & N-Acetyl-L-alanine & 1.63765 \\
\hline Uridine $5^{\prime}$-monophosphate & 1.07566 & Uridine $5^{\prime}$-monophosphate & 1.61569 \\
\hline Guanosine monophosphate & 1.038 & Hypoxanthine & 1.58986 \\
\hline Uric acid & 1.02329 & Sucrose & 1.57968 \\
\hline L-Histidine & 1.02007 & Acetoacetic acid & 1.57219 \\
\hline Inosinic acid & 1.00379 & L-Alanine & 1.44502 \\
\hline & & Betaine & 1.32999 \\
\hline & & Pyrrolidonecarboxylic acid & 1.29793 \\
\hline & & N-Acetyl-L-methionine & 1.21579 \\
\hline & & Pyridoxal & 1.15716 \\
\hline & & Azelaic acid & 1.11747 \\
\hline & & $\begin{array}{c}\text { Uridine } \\
\text { diphosphate-N-acetylglucosamine }\end{array}$ & 1.09991 \\
\hline & & L-Asparagine & 1.06611 \\
\hline & & Inosinic acid & 1.04028 \\
\hline & & Ophthalmic acid & 1.03219 \\
\hline & & Guanosine monophosphate & 1.03092 \\
\hline & & O-Phosphoethanolamine & 1.01278 \\
\hline & & L-Homoserine & 1.01177 \\
\hline & & Fumaric acid & 1.00205 \\
\hline
\end{tabular}

VIPs $>1$ found in PLS-DA-built models of CTRL vs. GLY comparison in (a) females and (b) males.

\subsection{Univariate Analysis to Identify Metabolic Profiles}

Those metabolites that following the PLS-DA model development, resulted were significant were used to perform univariate analysis on the MetaboAnalyst 5.0 online platform. In order to investigate changes at metabolite level (FDR $<0.05$ ), multiple t-tests were performed on CTRL and GLY-exposed groups using VIP $>1$ metabolites (GLY female $=12$; GLY male $=25$ ) in both male and female. To summarize the results, heatmaps were also provided, as shown in Figure 3a,b.

In female, exposure to GLY significantly increased sucrose, hypotaurine, L-homoserine, uric acid (UA), and docosahexaenoic acid (DHA), but decreased azelaic acid, adenosine monophosphate (AMP), uridine-5'-monophosphate (UMP), L-histidine (His), guanosine monophosphate (GMP), and inosinic acid (IMP) (Table 2a). 
a)

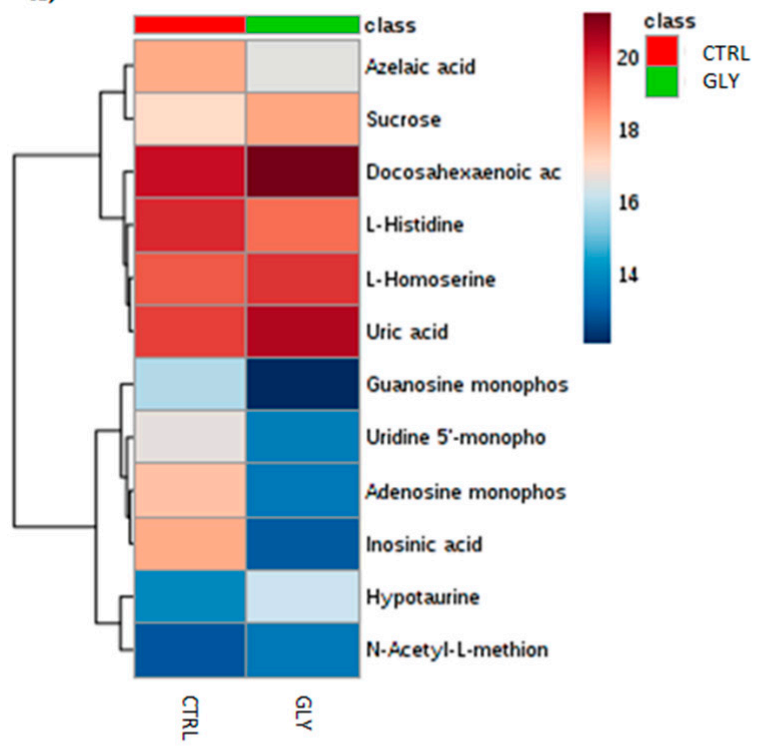

b)

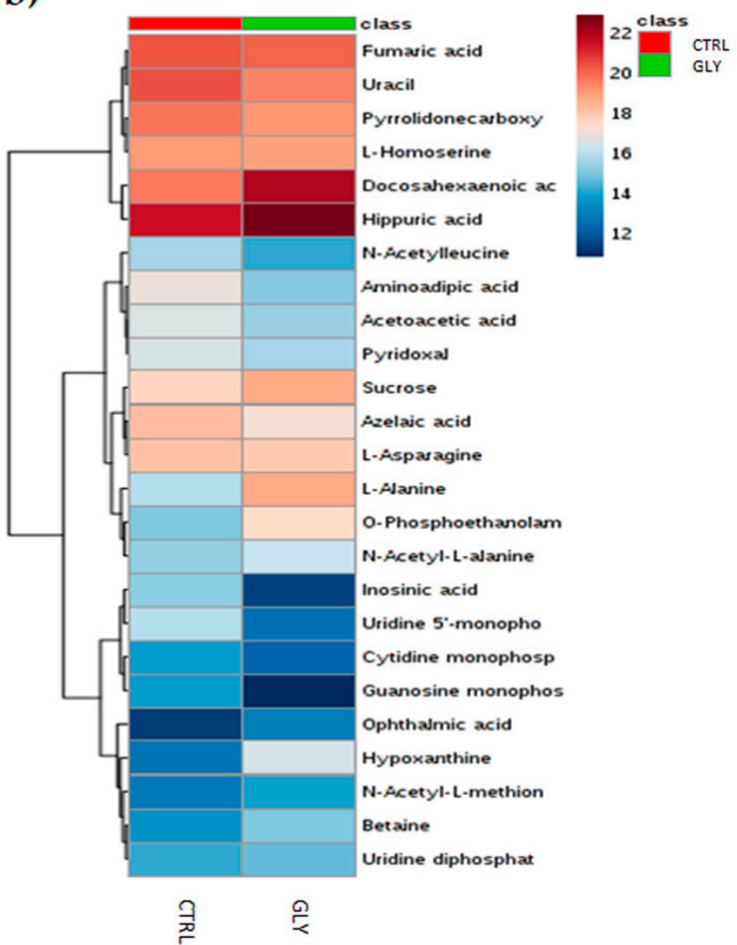

Figure 3. Clustered heatmap of VIP $>1$ in CTRL and GLY-exposed female and male livers. Heatmap showing the VIP $>1$ metabolites found in multivariate analysis (PLS-DA) of CTRL vs. GLY in (a) females and (b) males. Euclidean distance was used to perform clustering analysis. The heatmaps were constructed using Ward's algorithm and samples are shown as group average. Increase and decrease in metabolite concentration are indicated by red and blue shades, respectively; the more intense is the color, the greater the increase or the decrease.

Table 2. Metabolites modulated by GLY exposure in female and male livers.

\begin{tabular}{|c|c|c|c|c|c|c|c|}
\hline \multicolumn{3}{|c|}{ (a) } & & \multicolumn{4}{|c|}{ (b) } \\
\hline Metabolite Name & $p$-Value & FDR & & Metabolite Name & $p$-Value & FDR & \\
\hline Sucrose & 0.001231 & 0.008438 & $\uparrow$ & Aminoadipic acid & 0.000236 & 0.0017 & $\downarrow$ \\
\hline Azelaic acid & 0.001406 & 0.008438 & $\downarrow$ & & & & \\
\hline Hypotaurine & 0.002545 & 0.01018 & $\uparrow$ & & & & \\
\hline L-Homoserine & 0.005126 & 0.012943 & $\uparrow$ & & & & \\
\hline Uric acid & 0.005954 & 0.012943 & $\uparrow$ & & & & \\
\hline Adenosine monophosphate & 0.006472 & 0.012943 & $\downarrow$ & & & & \\
\hline Uridine $5^{\prime}$-monophosphate & 0.010393 & 0.015703 & $\downarrow$ & & & & \\
\hline L-Histidine & 0.010824 & 0.015703 & $\downarrow$ & & & & \\
\hline Docosahexaenoic acid & 0.011778 & 0.015703 & $\uparrow$ & & & & \\
\hline Guanosine monophosphate & 0.018083 & 0.019942 & $\downarrow$ & & & & \\
\hline Inosinic acid & 0.01828 & 0.019942 & $\downarrow$ & & & & \\
\hline
\end{tabular}

Multiple $t$-test analysis results showing metabolites significantly upregulated $(\uparrow)$ or down-regulated $(\downarrow)$ in CTRL vs. GLY comparison in (a) females and (b) males. $p$-value and FDR were reported; FDR $<0.05$ was considered significant.

Metabolomic pathway analysis (MetPA) is able to trace the involvment of metabolites within metabolic pathways. The analysis was performed using MetaboAnalyst 5.0 starting from the same dataset used for the multiple t-test. Sucrose was found to be involved in galactose, starch, and sucrose metabolism pathways. The AMP, IMP, GMP, and UA were involved in the purine metabolism pathway, while UMP was involved in the pyrimidine metabolism pathway. Hypotaurine was found in taurine and hypotaurine metabolism, DHA was found 
in the biosynthesis of unsaturated fatty acids and His in histidine and $\beta$-alanine metabolism as well as aminoacyl tRNA biosynthesis pathways (Table 3a and Figure 4a).

Table 3. Summary of the main altered metabolic pathways in fish liver exposed to GLY.

\begin{tabular}{|c|c|c|c|c|c|}
\hline \multicolumn{3}{|c|}{ (a) } & \multicolumn{3}{|c|}{ (b) } \\
\hline Pathway Name & FDR & Impact & Pathway Name & FDR & Impact \\
\hline Galactose metabolism & 0.005414 & 0.04029 & $\begin{array}{c}\text { Lysine } \\
\text { degradation }\end{array}$ & 0.0051841 & 0 \\
\hline $\begin{array}{l}\text { Starch and sucrose } \\
\text { metabolism }\end{array}$ & 0.005414 & 0.01534 & & & \\
\hline $\begin{array}{c}\text { Taurine and hypotaurine } \\
\text { metabolism }\end{array}$ & 0.0076346 & 0.4 & & & \\
\hline Purine metabolism & 0.011778 & 0.23384 & & & \\
\hline Pyrimidine metabolism & 0.011778 & 0.09066 & & & \\
\hline Histidine metabolism & 0.011778 & 0.2381 & & & \\
\hline Nitrogen metabolism & 0.011778 & 0 & & & \\
\hline $\begin{array}{l}\text { Aminoacyl-tRNA } \\
\text { biosynthesis }\end{array}$ & 0.011778 & 0 & & & \\
\hline $\begin{array}{l}\text { Biosynthesis of } \\
\text { unsaturated fatty acids }\end{array}$ & 0.011778 & 0 & & & \\
\hline
\end{tabular}

MetPA table showing main altered metabolic pathways found in CTRL vs. GLY comparison in (a) female and (b) male livers. FDR values $<0.05$ were considered statistically significantly altered and are shown. Impact is a value which refers to altered metabolite centrality in the changed pathways found.

a)

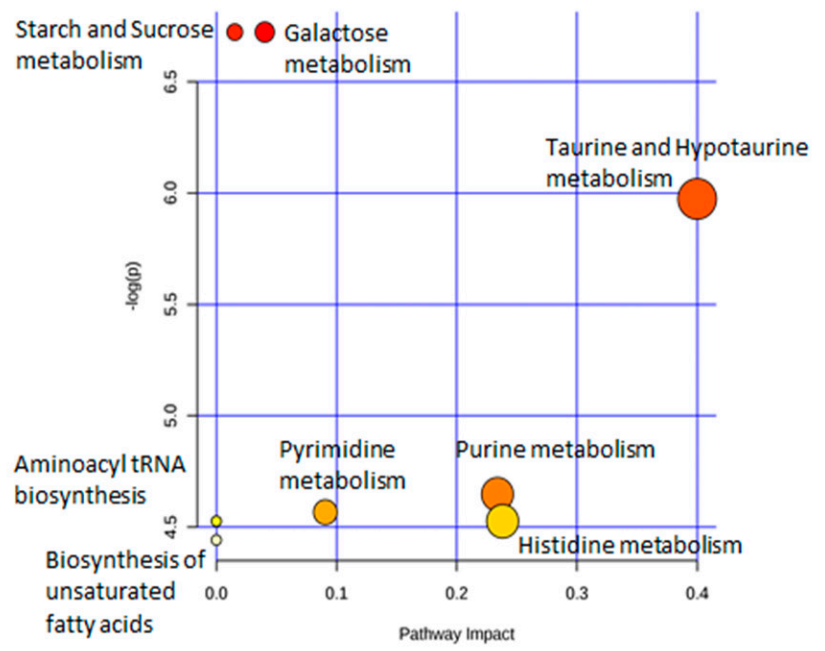

b)

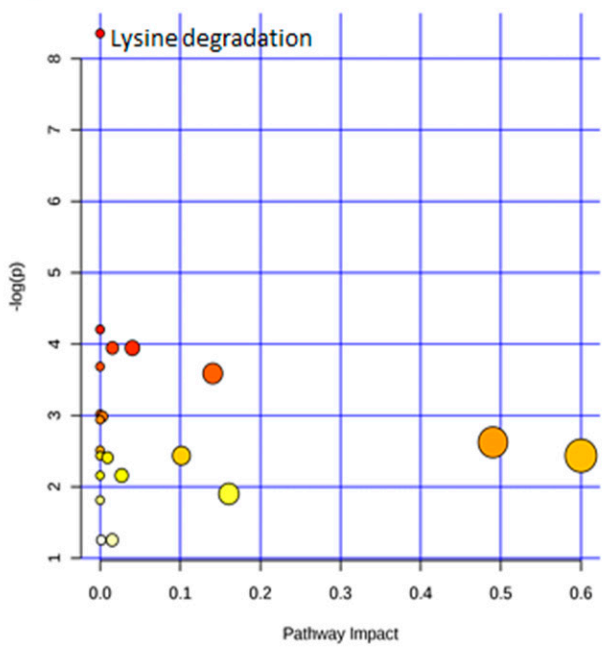

Figure 4. Dot plot of GLY-modulated pathways in the liver. MetPA graph showing main altered metabolic pathways found in CTRL vs. GLY comparison in (a) females and (b) males. The color of the dot (from white to red) reflects the significance $(F D R<0.05)$ of the pathway. The dimension of the dots reflects the impact of the metabolites in that pathway, the bigger the dot, the greater the impact of the metabolites in the pathway. The names of the pathways that were statistically significantly altered $(\mathrm{FDR}<0.05)$ are reported.

According to univariate analysis, only aminoadipic acid was significantly decreased in the GLY-exposed males (Table 2b), with MetPA identifying this metabolite inside the lysine degradation pathway (Table 3 b and Figure 4 b) which was the only pathway significantly affected. 


\subsection{Liver Transcript Analysis}

Based on transcript analysis, GLY exposure induced a different phenotype in females and males, while UA was upregulated in female livers. Considering that UA is able to induce oxidative stress when chronically elevated, genes involved in oxidative stress response were evaluated. Results clearly supported this hypothesis since GLY exposure significantly increased cat mRNA level in females, while in males, sod1, sod2, and gpx1a were reduced (Figure 5).

a)

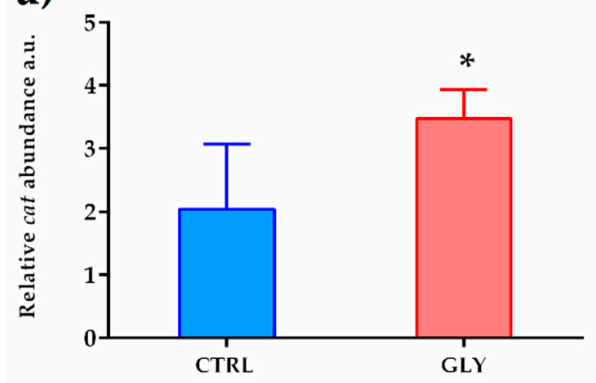

c)

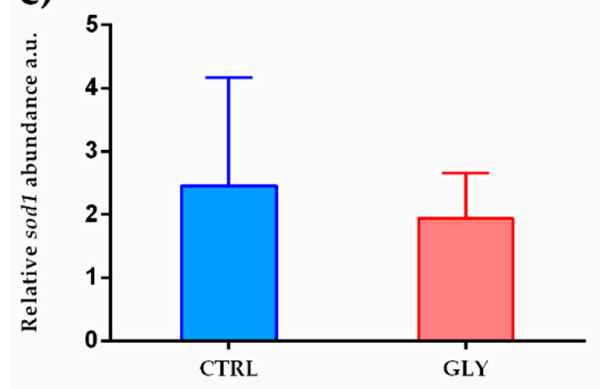

e)

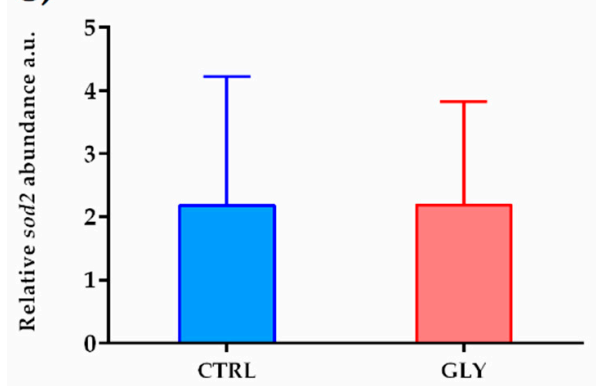

g)

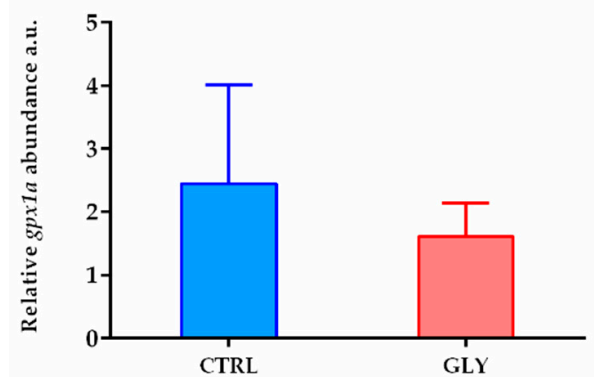

b)

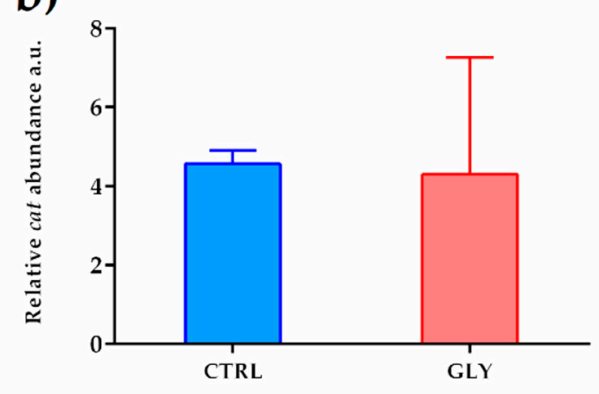

d)

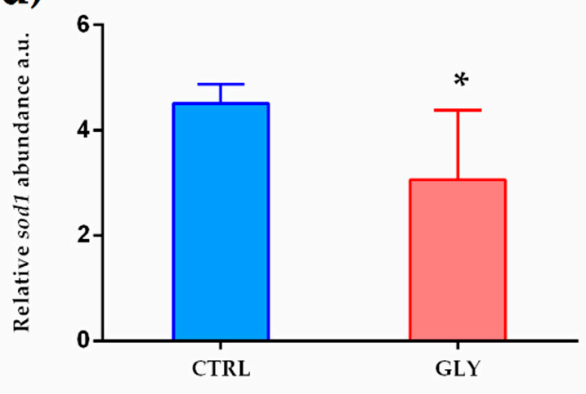

f)

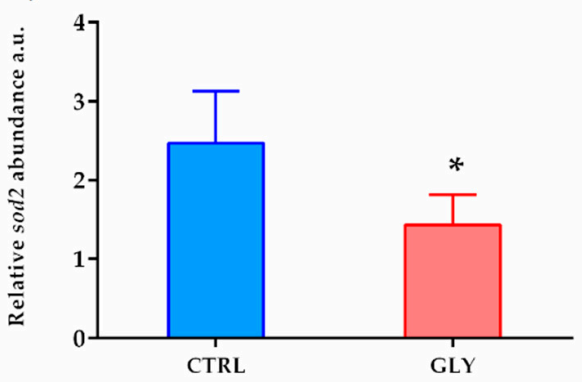

h)

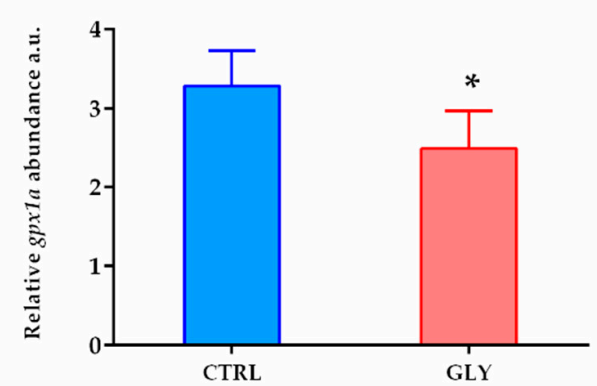

Figure 5. Gene expression profiles of hepatic oxidative stress response biomarkers. cat $(\mathbf{a}, \mathbf{b}) \operatorname{sod} 1(\mathbf{c}, \mathbf{d})$, sod2 (e,f), and gpx1a (g,h) mRNA levels in female and male livers, respectively, normalized against $r p l p 0$ and rplp13 in CTRL and GLY-exposed zebrafish. Data are shown as mean $\pm \mathrm{SD}(n=5)$ and were analyzed by the $t$-test. Asterisks above each column denote significant differences between the experimental groups $\left({ }^{*} p<0.05\right)$. 
Furthermore, GLY exposure increased $n r 3 c 1$ levels in both sexes, while $h s p 70.2$ transcript level was increased in females and decreased in males (Figure 6). These two genes, as stress response genes, were reliable biomarkers of GLY exposure and their levels provided information on the organism's oxidative-stress and immune-system responses.
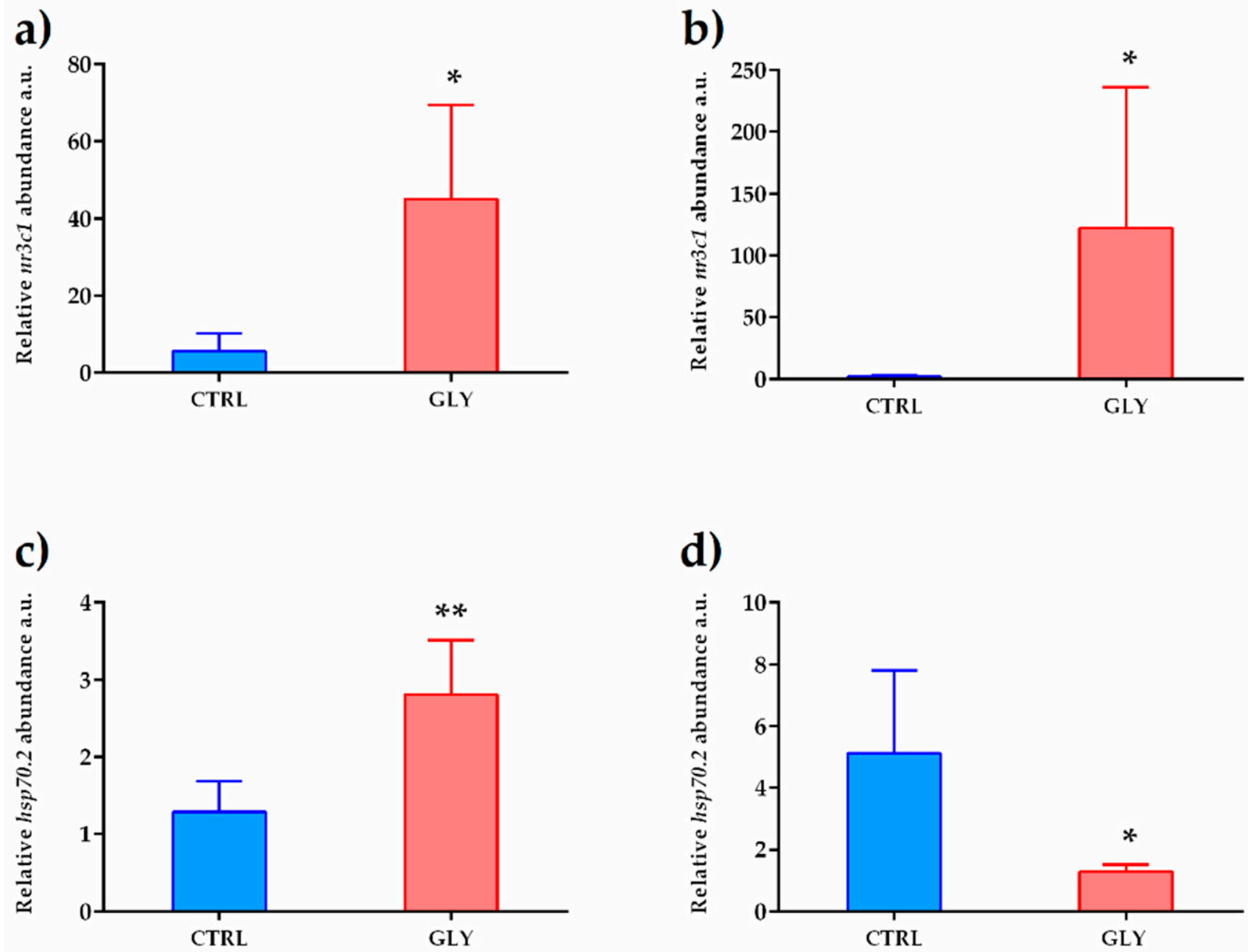

Figure 6. Gene-expression profile of stress-response biomarkers. $n r 3 c 1(\mathbf{a}, \mathbf{b})$ and $h s p 70.2(\mathbf{c}, \mathbf{d}) \mathrm{mRNA}$ levels in female and male livers, respectively, normalized against $r p l p 0$ and rplp13 in CTRL and GLY-exposed zebrafish. Data are shown as mean $\pm \mathrm{SD}(n=5)$ and were analyzed by the $t$-test. Asterisks above each column denote significant differences between the experimental groups $(* p<0.05$, ** $p<0.01$ ).

Since immune system and oxidative stress are related processes, a set of genes involved in the immune system response was evaluated. In males, no significant changes were found regarding those genes involved in immune response, while in females, levels of pro-inflammatory gene transcripts, interleukins litaf and cxcl8b.1, were increased (Figure 7).

Esr1 mRNA levels were further analyzed in female and male livers to compare differences in mRNA abundance between sexes. In females, esr1 mRNA levels were significantly higher than in male fish. Treatment did not affect basal mRNA levels in either males or females (Figure 8). 
a)

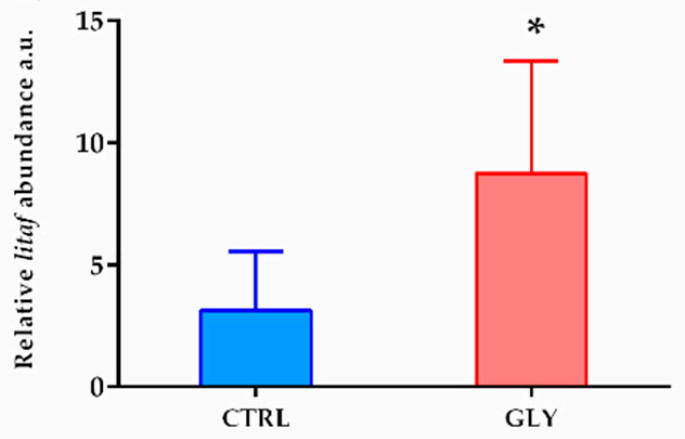

c)

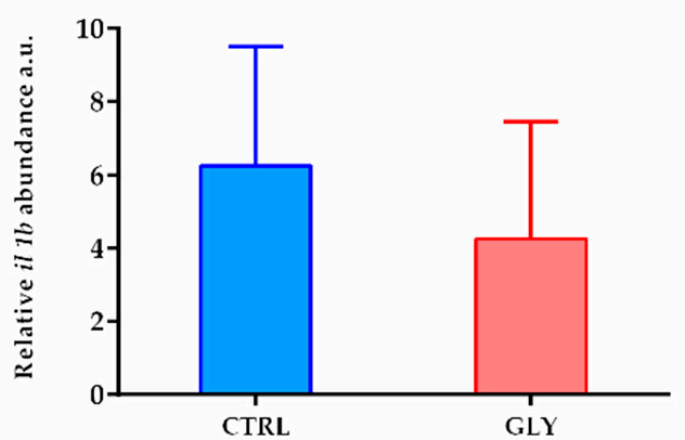

e)

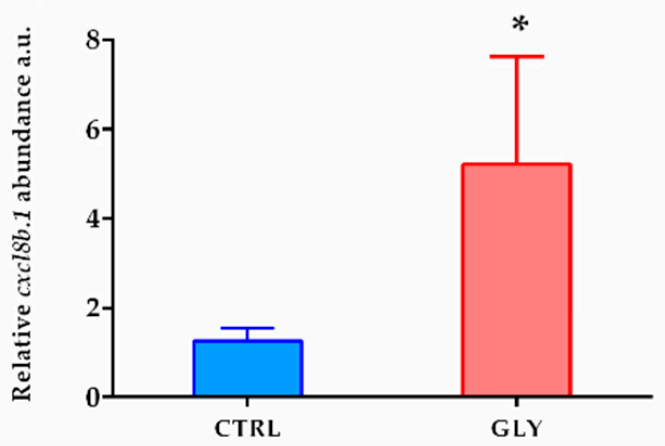

g)

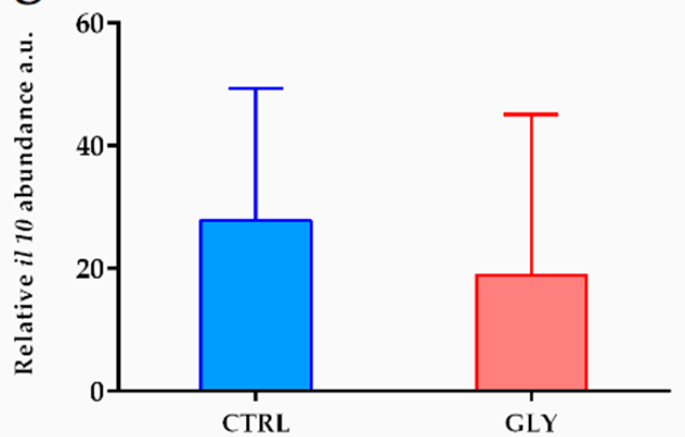

b)

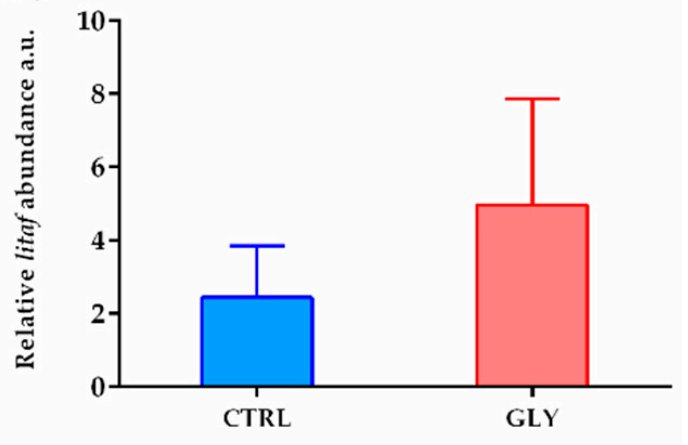

d)

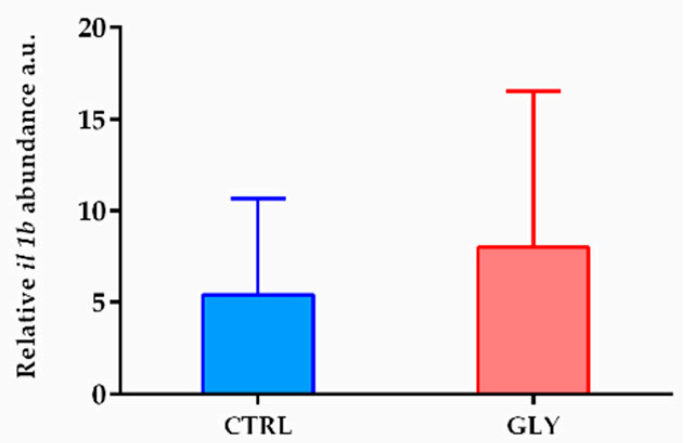

f)

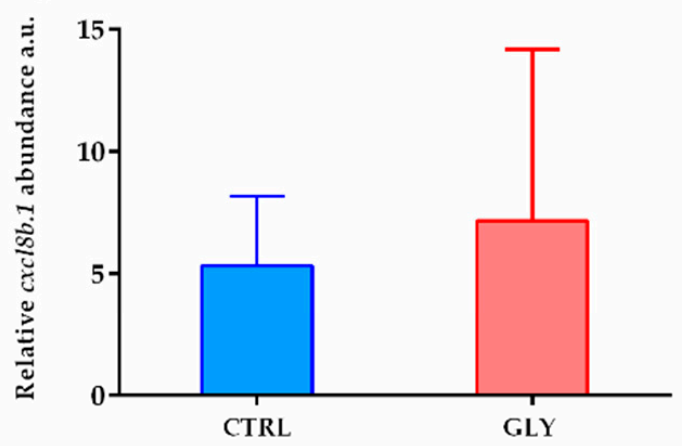

h)

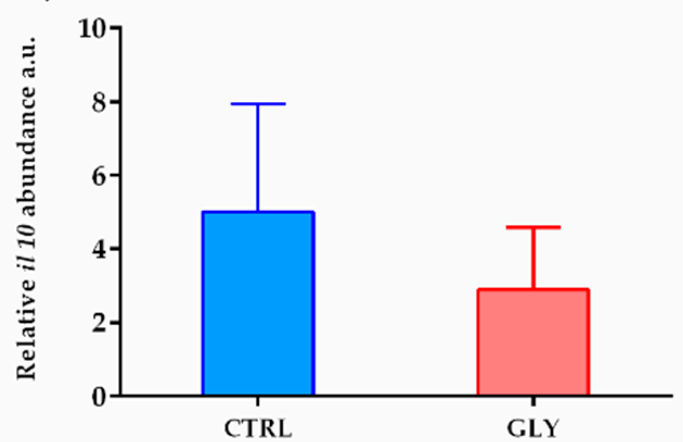

Figure 7. Gene expression profile of immune system biomarkers. litaf (a,b), il1b (c,d), cxcl8b.1 (e,f), and $i l 10(\mathbf{g}, \mathbf{h})$ mRNA levels in female and male livers, respectively, normalized against $r p l p 0$ and rplp13 in CTRL and GLY-exposed zebrafish. Data are shown as mean $\pm \mathrm{SD}(n=5)$ and were analyzed by the $t$-test. Asterisks above each column denote significant differences between the experimental groups $(* p<0.05)$. 


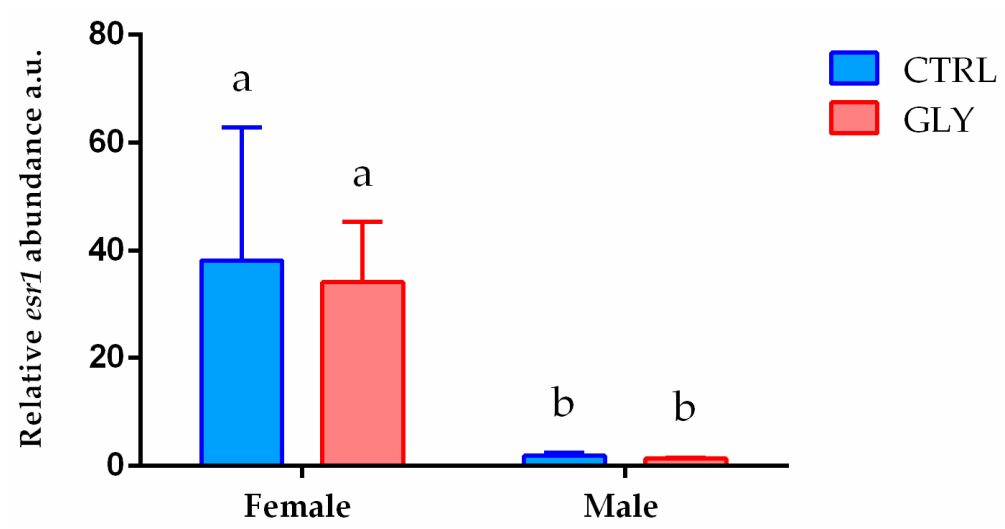

Figure 8. Gene expression profiles of hepatic esr1. esr1 mRNA levels in female and male livers normalized against rplp0 and rplp13 in CTRL and GLY-exposed zebrafish. Data are shown as mean \pm SD $(n=5)$ and were analyzed by two-way ANOVA followed by Tukey's multiple comparison test. Letters above each column denote significant differences among the experimental groups $(p<0.05)$.

\section{Discussion}

A metabolomic- and a transcriptomic-based approach was conducted on female and male zebrafish livers to investigate hepatic metabolic changes after chronic exposure to glyphosate. The initial experimental design also included one more group exposed to one dose of the commercial formulation Roundup ${ }^{\circledR}$, containing an equivalent concentration of glyphosate $(700 \mu \mathrm{g} / \mathrm{L})$. However, all fish died one hour after the addition of the Roundup. Likely, GLY alone did not cause mortality, and other compounds in the Roundup ${ }^{\circledR}$ made the mixture very toxic. Indeed, as previously demonstrated [12], the adverse effects of this herbicide on the aquatic ecosystem are attributable to the co-adjuvants.

Multivariate analysis of liver metabolism in females showed a significant shift in the GLY-treated fish compared to the CTRL fish. The univariate analysis revealed sex-specific differences in the GLY response, with females showing greater susceptibility to deleterious effects of the contaminant. In females, we observed disruption of purine metabolism, highlighted by reduction of purine intermediates AMP, IMP, and GMP, together with a decrease of the pyrimidine intermediate, UMP. Decrease of these metabolites could result from depletion of their precursor molecule, phosphoribosyl pyrophosphate (PRPP) [13]. The utilization of AMP, IMP, and GMP could lead to an increased level of UA which is the final product of the purine metabolism pathway, associated with increased oxidative stress [14]. Similar results were previously observed in goldfish, Carassius auratus, exposed to Nongteshi ${ }^{\circledR}$ [15] which is an herbicide containing 30\% $(w / v)$ GLY, leading to decreased inosine and GTP.

In the present study, we observed depletion of His in the liver of GLY-exposed females. There is evidence that the essential amino acid His has antioxidant, anti-inflammatory, and metal-ion chelation properties when supplied through the diet [16]. The primary source of His is the diet and protein turnover, and its metabolism leads to the production of carnosine, homocarnosine, histidine-rich protein, histamine, and glutamic acid [16]. His depletion observed in the female liver could be a contributing factor for oxidative stress induced by the contaminant. Other studies in fish demonstrated that a low level of His increases the tolerance to hypoxia [17], leading to stress conditions and the formation of ROS. Furthermore, His supplementation was observed to possess protective effects against liver injury caused by acetaminophen and ethanol in mice $[18,19]$ and may contribute to stress and anti-inflammatory response. Thus, the present study indicates that GLY-treated female fish are more susceptible to oxidative stress and ROS effects.

Hypotaurine, a sulfinic acid intermediate in taurine biosynthesis, is another metabolite possessing antioxidant capacity and acting as an osmolyte inside the cells [20,21]. The liver of GLY-exposed females contained an increased level of hypotaurine within the taurine and hypotaurine metabolism pathway. This is consistent with the reported increase level of 
taurine in Carassius auratus exposed to a GLY-based herbicide [15]. Furthermore, hepatic increase of this metabolite was also found in diet-induced rats with non-alcoholic fatty liver disease (NAFLD), a pathologic condition fostering inflammation and production of oxidative stress [22]. Studies concerning humans with acute liver failure demonstrated increased hypotaurine and decreased ATP together with lower levels of intermediates involved in glycolysis and pentose phosphate pathways [23]. The latter study indicates that, in case of liver abnormality, a hepatic shift from glycolysis to trans-sulfuration metabolism may occur. Similar conditions appear to be present in the liver of GLY- exposed female fish displaying a decrease in purine and pyrimidine intermediates and His levels which are derived from PRPP and the pentose phosphate pathway, together with increased hypotaurine levels. Furthermore, in GLY-exposed female fish, sucrose levels increased in connection with the starch, sucrose, and galactose metabolic pathways, suggesting a contaminant-induced reduction in the metabolism of sugars. Similar results were reported in goldfish treated with Nongteshi ${ }^{\circledR}$ [15], leading to decreased pyruvate kinase activity, a rate-limiting step glycolytic enzyme.

Exposure to GLY resulted in increased DHA in female fish, potentially impacting fatty acid metabolism. There is evidence that DHA reduces inflammatory and oxidative stress response [24] and liver fibrosis [25] in rats treated with the organophosphate chlorpyrifos or carbon tetrachloride, respectively. The enhancement of DHA and cat production in the liver of females exposed to GLY could be a possible mechanism to counteract GLY-induced hepatotoxicity and inflammation mediated by lifaf, cxcl8b.1 and interleukins.

Exposure of females to GLY also increased transcript levels of the genes involved in the stress response, including $n r 3 c 1$ and $h s p 70.2$, possibly mediated by glucocorticoid receptors [26-28]. The observed increase in the level of hsp70.2, which codes for HSP70, a heat shock protein chaperon of relevance in the fish cellular response to stress [29], provides further evidence that female zebrafish were responding to GLY-induced stress. In this context, $h s p 70.2$ expression is a reliable biomarker of xenobiotic exposure since it is upregulated by a different broad spectrum of pollutants [30-34]. Surprisingly, the GLY-exposed male metabolic phenotype was not significantly different from CTRL in the multivariate analysis. In this regard, only a decrease in aminoadipic acid levels in connection with the lysine degradation pathway was found to be significant. Aminoadipic acid is metabolized into glutaryl-CoA, which is then converted to acetyl-CoA and enters the citrate cycle. A possible explanation for the observed decrease of this metabolite is its enhanced catabolism to form intermediates used in energy production.

Decreased transcript levels of genes involved in oxidative stress response, including sod1, sod2, and gpx1a in GLY-exposed male fish, indicate a diminished capacity to counteract ROS-induced stress. The GLY-induced oxidative stress response was previously reported in rats $[3,35]$ and fish $[6,36]$. In the male liver, the expression of stress response genes showed an increased level of the glucocorticoid receptor with decreased $h s p 70.2$ level indicating that GLY exposure also affects the stress response, but in contrast to females, male fish may not be able to counteract the stress response using a similar mechanism to females. Our results are consistent with a previous study in Oreochromis niloticus chronically exposed to GLY [6].

There is clear evidence that GLY acts as an endocrine disruptor compound (EDC) [37]. This compound interacts with nuclear hormone receptors, including the estrogen receptor alpha $(E R \alpha)$. In this regard, GLY exposure enhanced ER $\alpha$ activation and stimulated its transcriptional activation in human breast cancer cells [37]. Furthermore, in vivo studies demonstrated the ability of GLY to alter hypothalamic gene expression, increase testosterone synthesis in the ovaries, and alter estradiol-sensitive genes in rats [37]. It is interesting that estradiol stimulation was observed to enhance amidophosphoribosyltransferase (PPAT) gene expression in breast cancer cells, which are involved in PRPP conversion to 5-phosphoribosyl-1-amine (PRA) for purine de novo synthesis [38]. By altering steroid hormone levels, it is possible to speculate that the decreased levels of purine intermediates are due to an impairment of this conversion through altered hormone homeostasis. 
Therefore, a possible explanation of the sex-specific effects observed in our study could be the capacity of GLY to interact with ER. In females, ERs are key receptors that maintain ovarian granulosa cell differentiation and follicle and oocyte growth and development [39]. However, beyond the canonical role in reproductive functions, estrogens also play a role in the regulation of other physiological functions such as immune response, growth, neuronal function, and metabolism [40-42]. Thus, by targeting estrogen receptors which are more abundant in females than in males, GLY could have different sex-related disruptive effects.

Overall, the present results provide evidence that chronic exposure to GLY dysregulates metabolism in zebrafish. We provide evidence that GLY exposure leads to stress response in Danio rerio in a sex-dependent manner. Our study also highlights the acute toxicity of GLY when formulated with coadjutants and surfactants such as commonly used GLY-based herbicides. Thus, it would be hard to suggest a safe environmental level for GLY as its toxicity changes significantly when combined with coadjutants. Further studies are needed to provide more evidence on the toxicity of GLY combined with coadjutants to better assess the risks associated with this type of herbicide.

\section{Materials and Methods}

\subsection{Animal Treatment and Maintenance}

In order to assess GLY toxicity, 90 adult zebrafish (D. rerio, AB wild-type strain) were divided into four aquaria of $20 \mathrm{~L}$ each ( $20 \mathrm{fish} / \mathrm{tank}$ ), in duplicate, under controlled conditions $\left(28.0 \pm 0.5^{\circ} \mathrm{C}\right)$ and with a $14 / 10 \mathrm{~h} \mathrm{light/dark}$ cycle in oxygenated water. Fish were fed twice a day with commercial food (TetraMin Granules; Tetra, Melle, Germany) in the morning and Artemia salina in the afternoon, with a quantity of food ranging from $2.5 \%$ to $3.0 \%$ of fish body weight. Water was changed every two days. Two experimental groups were set up as follow:

Control (CTRL): fish fed twice a day with commercial food (TetraMin Granules; Tetra, Melle, Germany) and Artemia salina.

Glyphosate (GLY): fish were fed commercial food and Artemia salina, and were exposed to $700 \mu \mathrm{g} / \mathrm{L}$ of glyphosate (98\% analytical purity, Sigma-Aldrich, Milano, Italy) via water (GLY powder was dissolved in water before administration every two days.

All groups were sampled after 4 weeks of treatment. Fish were euthanized with $500 \mathrm{mg} / \mathrm{L}$ MS-222 (3-aminobenzoic acid ethyl ester, Sigma Aldrich) buffered to $\mathrm{pH}$ 7.4. For metabolomic analysis livers were pooled (three livers per pool), while for transcript analysis five livers were sampled. All samples were stored at $-80{ }^{\circ} \mathrm{C}$ until processed for analysis. Procedures involving animals were conducted following the University of Calgary animal care protocol (AC16-0160) for care and use of experimental animals, all efforts were made to minimize suffering.

\subsection{Metabolite Extraction and UHPLC-ESI-MS Analysis}

Liver metabolite extraction, separation, and identification were performed on seven pools of female livers and four pools of male livers for each investigated group, as well as the quality control group $(\mathrm{QC} ; n=4)$ generated by pooling random samples as described previously [43]. The number of pools analyzed was determined on the basis of the fish sex ratio while sampling. Effort was made to analyze the same number of pools between CTRL and GLY females or males. Liver samples $(20 \mathrm{mg})$ were homogenized using a beadbeating homogenizer (TissueLyser II, QIAGEN) and extracted with methanol (ratio of 1:20 sample $(\mathrm{mg})$ : methanol $(\mu \mathrm{L})$ ). After $20 \mathrm{~min}$ of centrifugation at 13,500 rpm, the supernatant was collected and stored at $-80{ }^{\circ} \mathrm{C}$ until further analysis. The quality control group (QC) was generated by pooling $20 \mathrm{mg}$ from five random samples, extracted following the same protocol and analyzed in four statistical replicates. Metabolites were analyzed using ultra-high-performance liquid chromatography (UHPLC) mass spectrometry (MS), with a hydrophilic-interaction liquid chromatography column (Syncronis HILIC, Thermo Fisher, stationary phase) to separate the metabolites. High-resolution full-scan MS data were acquired on a Thermo Fisher Scientific Q-Exactive HF mass spectrometer using negative- 
mode electrospray ionization. MAVEN freeware and an $\mathrm{m} / \mathrm{z}$ and retention time standard were used for targeted profiling of the MS spectra and identification of the metabolites. Peak intensity was measured as area top, representing the average intensity of the top three points of the peak [43].

\subsection{RNA Extraction and cDNA Synthesis}

Extraction of total RNA was performed from five female and five male livers for CTRL and GLY experimental groups, using the RNAeasy ${ }^{\circledR}$ Minikit (Qiagen, Milano, Italy) following the manufacturer's instructions, and eluted in RNase-free water at a final volume of $50 \mu \mathrm{L}$. RNA quality evaluation and cDNA synthesis were assessed as previously described [44] and are here briefly reported. RNA concentration was determined using a NanophotometerTMP-Class (Implen GmbH, Munich, Germany), and $28 \mathrm{~S}$ and $18 \mathrm{~S}$ ribosomal RNA was stained using ethidium bromide on $1 \%$ agarose gel to determine RNA integrity.

DNase treatment (10 IU at $37^{\circ} \mathrm{C}$ for $10 \mathrm{~min}$, MBI Fermentas, Milano, Italy) was performed on total RNA to digest genomic DNA, and $1 \mu \mathrm{g}$ RNA was used for cDNA synthesis performed using the iScript cDNA Synthesis Kit (Bio-Rad, Milano, Italy) according to the manufacturer's instructions.

\subsection{Real-Time PCR}

In order to perform qRT-PCRs, a CFX thermal cycler was used with SYBR green, as described in [44], and ribosomal protein 13 (rpl13) and ribosomal protein $0(r p l p 0)$ mRNAs were used as internal standards in each sample to standardize the results and eliminate variation in mRNA and cDNA quantity and quality. Table 4 reports primer sequences, GenBank accession numbers, and primer efficiency of the examined genes.

Table 4. Primer list.

\begin{tabular}{|c|c|c|c|c|}
\hline Name & Forword & Reverse & Efficiency (Tm ${ }^{\circ}$ ) & Accession Number \\
\hline cat & CCAAGGTCTGGTCCCATAAA & GCACATGGGTCCATCTCTC & $60^{\circ}$ & NM_130912 \\
sod 1 & GTCGTCTGGCTTGTGGAGTG & TGTCAGCGGGCTAGTGCTT & $60^{\circ}$ & NM_131294 \\
sod 2 & CCGGACTATGTTAAGGCCATCT & ACACTCGGTTGCTCTCTTTTCTCT & $60^{\circ}$ & NM_199976 \\
gp $x 1 a$ & ACCTGTCCGCGAAACTATTG & TGACTGTTGTGCCTCAAAG & $59^{\circ}$ & NM_001007281.2 \\
$n r 3 c 1$ & CGCCTTTAATCATGGGAGAA & AGACCTTGGTCCCCTTCACT & $58^{\circ}$ & NM_001020711.3 \\
$h s p 70.2$ & TGTTCAGTTCTCTGCCGTTG & AAAGCACTGAGGGACGCTAA & $58^{\circ}$ & NM_001362360.1 \\
$l i t a f$ & TTGTGGTGGGGTTTGATG & TTGGGGCATTTTATTTTGTAAG & $53^{\circ}$ & NM_001002184.1 \\
$i l 1 b$ & GTGGATTGGGGTTTGATGTG & GCTGGGGATGTGGACTTC & $54^{\circ}$ & NM_212844.2 \\
$c x c l s b .1$ & ACTCGGACTGAAGGTGACTC & CCACGTCTCGGTAGGATTGAG & $58^{\circ}$ & NM_001327985 \\
$i l 10$ & GCCGTGGAGCAGGTGAAG & GAAGATGTCAAACTCACTCATGGCT & $58^{\circ}$ & NM_001020785 \\
esr 1 & GGTCCAGTGTGGTGTCCTCT & AGAAAGCTTTGCATCCCTCA & $58^{\circ}$ & NM_152959.1 \\
$r p l 13 a$ & TCTGGAGACTGTAAGAGGTATGC & AGACGCACAATCTTGAGAGCAG & $59^{\circ}$ & NM_198143 \\
$r p l p 0$ & CTGAACATCTCGCCCTTCTC & TAGCCGATCTGCAGACACAC & $60^{\circ}$ & NM_131580 \\
\hline
\end{tabular}

Furthermore, the calculation of mRNA levels of target genes analyzed was performed using the geometric mean of the two reference genes after demonstrating that they were stably expressed by the geNorm algorithm, both applications implemented in the Bio-Rad CFX Manager 3.1. software. Gene transcript expression alterations among experimental groups are reported as relative mRNA abundance (arbitrary units). Primers were used at a final concentration of $10 \mathrm{pmol} / \mathrm{mL}$.

\subsection{Statistical Analysis}

\subsubsection{Metabolomic Statistical Analysis}

Normalization, transformation, and scaling (normalization by a median, log transformation, and pareto scaling) were conducted prior to conducting multivariate analysis. SIMCA software (Umetrics, Umeå, Sweden), was used for partial least squares-discriminant analysis (PLS-DA) and hierarchical analysis model generation. Quality assessment of the 
models was indicated by R2 and Q2 $>0.5$, and models with $p<0.05$ were considered significant [43]. The MetaboAnalyst 5.0 online platform (University of Aberta, Alberta Canada) was used to perform unsupervised principal component analysis (PCA) on all treatments and QC to identify potential outliers and assess the LC-MS reliability. QC samples are usually some random samples that are pooled and extracted together. This extract is then aliquoted in different wells of the 96-well plate and analyzed before and after the samples of interest as technical replicates, to confirm the reliability of the technique.

Hierarchical cluster analysis was performed in a "bottom-up" manner using the Ward clustering algorithm. VIP (variable importance in projection) score $>1$ was used as a threshold for the identification of significant metabolites in each PLS-DA model. VIP $>1$ was used for hierarchical clustering (Ward clustering, Euclidean distance), univariate analysis t-test, and pathway analysis using Metaboanalyst 5.0 as described before [43]. A significant threshold of $p$-value adjusted using false discovery rate (FDR) $<0.05$ was used to assess statistically significant differences among experimental groups.

\subsubsection{Pathway Analysis}

Metabolites with VIP score $>1$ in each OPLS-DA model, were used to perform pathway analysis (MetPA) using the Metaboanalyst 5.0 platform [43]. This technique considers the concentration of each metabolite, using quantitative enrichment analysis (QEA) and the position in the pathway, using topological analysis. For these two parameters, global test and relative-betweenness centrality algorithms were selected, respectively, using the KEGG pathway library of zebrafish (Danio rerio) as reference.

\subsubsection{Gene Expression Statistical Analysis}

T-test analysis was performed to analyze transcript expression. Statistical software package Prism5 (GraphPad Software, Inc., San Diego, CA, USA) was used for statistical analyses with $p<0.05$ as threshold for statistical significance.

Author Contributions: Formal analysis, C.G., C.L. and F.M.; conceptualization, O.C. and H.R.H.; validation C.G., F.M. and C.L.; methodology, O.C., F.M., H.R.H., C.L. and C.G.; writing—original draft preparation C.G. and F.M.; writing-review and editing, F.M., C.G., H.R.H. and O.C. All authors have read and agreed to the published version of the manuscript.

Funding: This work was supported by the funding from Natural Sciences and Engineering Research Council of Canada to H.R.H. (NSERC Discovery Grant; project no. 1254045) and by Fondi di Ateneo, Università Politecnica delle Marche to O.C. C.L. was supported by NSERC grants to H.H. C.L. was also supported by the Alberta Graduate Excellence Scholarship (AGES).

Institutional Review Board Statement: The study was conducted according to the guidelines of the Declaration of Helsinki and was approved by the Ethics Committee of the University of CalgaryAnimal Care protocol (AC16-0160).

Informed Consent Statement: Not applicable.

Data Availability Statement: Not applicable.

Acknowledgments: Metabolomics data were acquired at the Calgary Metabolomics Research Facility (CMRF), which is supported by grants to Ian A. Lewis including the International Microbiome Centre, Canada Foundation for Innovation (CFI-JELF\#34986), the Alberta Innovates Translational Health Chair, and NSERC-DG \# 04547. The authors thank Ian Lewis for his expertise in metabolomics and the members of the University of Calgary's Metabolomics Research Facility for the technical support.

Conflicts of Interest: The authors declare no conflict of interest. 


\section{References}

1. Sikorski, J.A.; Gruys, K.J. Understanding Glyphosate's Molecular Mode of Action with EPSP Synthase: Evidence Favoring an Allosteric Inhibitor Model. Acc. Chem. Res. 1997, 30, 2-8. [CrossRef]

2. Forner-Piquer, I.; Faucherre, A.; Byram, J.; Blaquiere, M.; de Bock, F.; Gamet-Payrastre, L.; Ellero-Simatos, S.; Audinat, E.; Jopling, C.; Marchi, N. Differential impact of dose-range glyphosate on locomotor behavior, neuronal activity, glio-cerebrovascular structures, and transcript regulations in zebrafish larvae. Chemosphere 2021, 267, 128986. [CrossRef] [PubMed]

3. Milić, M.; Žunec, S.; Micek, V.; Kašuba, V.; Mikolić, A.; Lovaković, B.T.; Semren, T.Ž.; Pavičić, I.; Čermak, A.M.M.; Pizent, A.; et al. Oxidative stress, cholinesterase activity, and DNA damage in the liver, whole blood, and plasma of Wistar rats following a 28-day exposure to glyphosate. Arh. Hig. Rada Toksikol. 2018, 69, 154-168. [CrossRef]

4. Mutwedu, V.B.; Nyongesa, A.W.; Azine, P.C.; Chiregereza, D.K.; Ngoumtsop, V.H.; Mugumaarhahama, Y.; Ayagirwe, R.B.B. Growth performance and reproductive function impairment of glyphosate-based herbicide in male guinea pig (Cavia porcellus). Vet. Med. Sci. 2021, 7, 1047-1055. [CrossRef]

5. Mesnage, R.; Renney, G.; Séralini, G.E.; Ward, M.; Antoniou, M.N. Multiomics reveal non-alcoholic fatty liver disease in rats following chronic exposure to an ultra-low dose of Roundup herbicide. Sci. Rep. 2017, 7, 39328. [CrossRef] [PubMed]

6. Zheng, T.; Jia, R.; Cao, L.; Du, J.; Gu, Z.; He, Q.; Xu, P.; Yin, G. Effects of chronic glyphosate exposure on antioxdative status, metabolism and immune response in tilapia (GIFT, Oreochromis niloticus). Comp. Biochem. Physiol. Part C Toxicol. Pharmacol. 2021, 239, 108878. [CrossRef]

7. Le Du-Carrée, J.; Saliou, F.; Cachot, J.; Morin, T.; Danion, M. Developmental effect of parental or direct chronic exposure to environmental concentration of glyphosate on the larvae of rainbow trout, Oncorhynchus mykiss. Aquat. Toxicol. 2021, 237, 105894. [CrossRef]

8. Le Du-Carrée, J.; Boukhari, R.; Cachot, J.; Cabon, J.; Louboutin, L.; Morin, T.; Danion, M. Generational effects of a chronic exposure to a low environmentally relevant concentration of glyphosate on rainbow trout, Oncorhynchus mykiss. Sci. Total Environ. 2021, 801, 149462. [CrossRef]

9. Du-Carrée, J.L.; Morin, T.; Danion, M. Impact of chronic exposure of rainbow trout, Oncorhynchus mykiss, to low doses of glyphosate or glyphosate-based herbicides. Aquat. Toxicol. 2021, 230, 105687. [CrossRef]

10. Weeks Santos, S.; Gonzalez, P.; Cormier, B.; Mazzella, N.; Bonnaud, B.; Morin, S.; Clérandeau, C.; Morin, B.; Cachot, J. A glyphosate-based herbicide induces sub-lethal effects in early life stages and liver cell line of rainbow trout, Oncorhynchus mykiss. Aquat. Toxicol. 2019, 216, 105291. [CrossRef]

11. Gillezeau, C.; Van Gerwen, M.; Shaffer, R.M.; Rana, I.; Zhang, L.; Sheppard, L.; Taioli, E. The evidence of human exposure to glyphosate: A review. Environ. Health 2019, 18, 2. [CrossRef] [PubMed]

12. Van Bruggen, A.H.C.; He, M.M.; Shin, K.; Mai, V.; Jeong, K.C.; Finckh, M.R.; Morris, J.G. Environmental and health effects of the herbicide glyphosate. Sci. Total Environ. 2018, 616-617, 255-268. [CrossRef] [PubMed]

13. Hove-Jensen, B.; Andersen, K.R.; Kilstrup, M.; Martinussen, J.; Switzer, R.L.; Willemoës, M. Phosphoribosyl Diphosphate (PRPP): Biosynthesis, Enzymology, Utilization, and Metabolic Significance. Microbiol. Mol. Biol. Rev. 2016, 81, e00040-16. [CrossRef] [PubMed]

14. George, C.; Minter, D.A. Hyperuricemia. In Urology at a Glance; Springer: Berlin/Heidelberg, Germany, 2021; pp. 107-109. [CrossRef]

15. Li, M.H.; Ruan, L.Y.; Zhou, J.W.; Fu, Y.H.; Jiang, L.; Zhao, H.; Wang, J.S. Metabolic profiling of goldfish (Carassius auratis) after long-term glyphosate-based herbicide exposure. Aquat. Toxicol. 2017, 188, 159-169. [CrossRef] [PubMed]

16. Holeček, M. Histidine in Health and Disease: Metabolism, Physiological Importance, and Use as a Supplement Milan. Nutrients 2020, 12, 848. [CrossRef]

17. Gao, Y.J.; Liu, Y.J.; Chen, X.Q.; Yang, H.J.; Li, X.F.; Tian, L.X. Effects of graded levels of histidine on growth performance, digested enzymes activities, erythrocyte osmotic fragility and hypoxia-tolerance of juvenile grass carp Ctenopharyngodon idella. Aquaculture 2016, 452, 388-394. [CrossRef]

18. Liu, W.-h.; Liu, T.-c.; Yin, M. chin Beneficial effects of histidine and carnosine on ethanol-induced chronic liver injury. Food Chem. Toxicol. 2008, 46, 1503-1509. [CrossRef]

19. Yan, S.L.; Wu, S.T.; Yin, M.C.; Chen, H.T.; Chen, H.C. Protective effects from carnosine and histidine on acetaminophen-induced liver injury. J. Food Sci. 2009, 74, H259-H265. [CrossRef]

20. Baseggio Conrado, A.; D'Angelantonio, M.; D'Erme, M.; Pecci, L.; Fontana, M. The Interaction of Hypotaurine and Other Sulfinates with Reactive Oxygen and Nitrogen Species: A Survey of Reaction Mechanisms. Adv. Exp. Med. Biol. 2017, 975, 573-583. [CrossRef]

21. Yancey, P.H. Organic osmolytes as compatible, metabolic and counteracting cytoprotectants in high osmolarity and other stresses. J. Exp. Biol. 2005, 208, 2819-2830. [CrossRef]

22. Kwon, D.Y.; Jung, Y.S.; Kim, S.J.; Park, H.K.; Park, J.H.; Kim, Y.C. Impaired sulfur-amino acid metabolism and oxidative stress in nonalcoholic fatty liver are alleviated by betaine supplementation in rats. J. Nutr. 2009, 139, 63-68. [CrossRef] [PubMed]

23. Mizota, T.; Hishiki, T.; Shinoda, M.; Naito, Y.; Hirukawa, K.; Masugi, Y.; Itano, O.; Obara, H.; Kitago, M.; Yagi, H.; et al. The hypotaurine-taurine pathway as an antioxidative mechanism in patients with acute liver failure. J. Clin. Biochem. Nutr. 2022, 70, 54-63. [CrossRef] [PubMed] 
24. Avci, B.; Bilge, S.S.; Arslan, G.; Alici, O.; Darakci, O.; Baratzada, T.; Ciftcioglu, E.; Yardan, T.; Bozkurt, A. Protective effects of dietary omega-3 fatty acid supplementation on organophosphate poisoning. Toxicol. Ind. Health 2018, 34, 69-82. [CrossRef] [PubMed]

25. He, J.; Bai, K.; Hong, B.; Zhang, F.; Zheng, S. Docosahexaenoic acid attenuates carbon tetrachloride-induced hepatic fibrosis in rats. Int. Immunopharmacol. 2017, 53, 56-62. [CrossRef]

26. Malandrakis, E.E.; Dadali, O.; Golomazou, E.; Kavouras, M.; Dailianis, S.; Chadio, S.; Exadactylos, A.; Panagiotaki, P. DNA damage and differential gene expression associated with physical stress in gilthead seabream (Sparus aurata). Gen. Comp. Endocrinol. 2016, 236, 98-104. [CrossRef]

27. Alves, R.N.; Cordeiro, O.; Silva, T.S.; Richard, N.; de Vareilles, M.; Marino, G.; Di Marco, P.; Rodrigues, P.M.; Conceição, L.E.C. Metabolic molecular indicators of chronic stress in gilthead seabream (Sparus aurata) using comparative proteomics. Aquaculture 2010, 299, 57-66. [CrossRef]

28. Traversi, I.; Gioacchini, G.; Scorolli, A.; Mita, D.G.; Carnevali, O.; Mandich, A. Alkylphenolic contaminants in the diet: Sparus aurata juveniles hepatic response. Gen. Comp. Endocrinol. 2014, 205, 185-196. [CrossRef]

29. Stankiewicz, A.R.; Lachapelle, G.; Foo, C.P.Z.; Radicioni, S.M.; Mosser, D.D. Hsp70 inhibits heat-induced apoptosis upstream of mitochondria by preventing Bax translocation. J. Biol. Chem. 2005, 280, 38729-38739. [CrossRef]

30. Migliarini, B.; Campisi, A.M.; Maradonna, F.; Truzzi, C.; Annibaldi, A.; Scarponi, G.; Carnevali, O. Effects of cadmium exposure on testis apoptosis in the marine teleost Gobius niger. Gen. Comp. Endocrinol. 2005, 142, 241-247. [CrossRef]

31. Carnevali, O.; Maradonna, F. Exposure to xenobiotic compounds: Looking for new biomarkers. Gen. Comp. Endocrinol. 2003, 131, 203-208. [CrossRef]

32. Maradonna, F.; Carnevali, O. Vitellogenin, zona radiata protein, cathepsin D and heat shock protein 70 as biomarkers of exposure to xenobiotics. Biomarkers 2007, 12, 240-255. [CrossRef] [PubMed]

33. Randazzo, B.; Chemello, G.; Tortarolo, I.; Chiarello, G.L.; Zalas, M.; Santini, A.; Liberatore, M.; Liberatore, M.; Selli, E.; Olivotto, I. A Novel Photocatalytic Purification System for Fish Culture. Zebrafish 2017, 14, 411-421. [CrossRef] [PubMed]

34. Gioacchini, G.; Giorgini, E.; Olivotto, I.; Maradonna, F.; Merrifield, D.L.; Carnevali, O. The influence of probiotics on zebrafish Danio rerio innate immunity and hepatic stress. Zebrafish 2014, 11, 98-106. [CrossRef]

35. Soudani, N.; Chaâbane, M.; Ghorbel, I.; Elwej, A.; Boudawara, T.; Zeghal, N. Glyphosate disrupts redox status and up-regulates metallothionein I and II genes expression in the liver of adult rats. Alleviation by quercetin. Gen. Physiol. Biophys. 2019, 38, 123-134. [CrossRef] [PubMed]

36. Modesto, K.A.; Martinez, C.B.R. Roundup causes oxidative stress in liver and inhibits acetylcholinesterase in muscle and brain of the fish Prochilodus lineatus. Chemosphere 2010, 78, 294-299. [CrossRef] [PubMed]

37. Muñoz, J.P.; Bleak, T.C.; Calaf, G.M. Glyphosate and the key characteristics of an endocrine disruptor: A review. Chemosphere 2021, 270, 128619. [CrossRef]

38. Zhu, D.; Zhao, Z.; Cui, G.; Chang, S.; Hu, L.; See, Y.X.; Lim, M.G.L.; Guo, D.; Chen, X.; Robson, P.; et al. Single-Cell Transcriptome Analysis Reveals Estrogen Signaling Coordinately Augments One-Carbon, Polyamine, and Purine Synthesis in Breast Cancer. Cell Rep. 2018, 25, 2285-2298.e4. [CrossRef]

39. Tang, Z.R.; Zhang, R.; Lian, Z.X.; Deng, S.L.; Yu, K. Estrogen-Receptor Expression and Function in Female Reproductive Disease Cells 2019, 8, 1123. [CrossRef]

40. Hussain, Y.; Ding, Q.; Connelly, P.W.; Brunt, J.H.; Ban, M.R.; McIntyre, A.D.; Huff, M.W.; Gros, R.; Hegele, R.A.; Feldman, R.D. G-protein estrogen receptor as a regulator of low-density lipoprotein cholesterol metabolism: Cellular and population genetic studies. Arterioscler. Thromb. Vasc. Biol. 2015, 35, 213-221. [CrossRef]

41. Avtanski, D.; Novaira, H.J.; Wu, S.; Romero, C.J.; Kineman, R.; Luque, R.M.; Wondisford, F.; Radovick, S. Both estrogen receptor $\alpha$ and $\beta$ stimulate pituitary GH gene expression. Mol. Endocrinol. 2014, 28, 40-52. [CrossRef]

42. Zhang, Z.C.; Liu, Y.; Xiao, L.L.; Li, S.F.; Jiang, J.H.; Zhao, Y.; Qian, S.W.; Tang, Q.Q.; Li, X. Upregulation of miR-125b by estrogen protects against non-alcoholic fatty liver in female mice. J. Hepatol. 2015, 63, 1466-1475. [CrossRef] [PubMed]

43. Ladisa, C.; Ma, Y.; Habibi, H.R. Seasonally related metabolic changes and energy allocation associated with growth and reproductive phases in the liver of male goldfish (Carassius auratus). J. Proteomics 2021, 241, 104237. [CrossRef] [PubMed]

44. Giommi, C.; Habibi, H.R.; Candelma, M.; Carnevali, O.; Maradonna, F. Probiotic Administration Mitigates Bisphenol A Reproductive Toxicity in Zebrafish. Int. J. Mol. Sci. 2021, 22, 9314. [CrossRef] [PubMed] 